\title{
Probabilistic Elastic-Plastic Cam Clay Response of Soils
}

\author{
Arezoo Sadrinezhad ${ }^{1}$, Kallol Sett ${ }^{2}$ \\ ${ }^{1}$ California State University Fresno \\ 2320 E. San Ramon Ave., Fresno, USA \\ ${ }^{2}$ The State University of New York at Buffalo \\ 221 Ketter Hall, Buffalo, USA \\ asadrinezhad@csufresno.edu; kallolse@buffalo.edu
}

\begin{abstract}
This study uses Cam Clay model to investigate the influence of uncertainties in soil properties on simulated undrained triaxial constitutive response. To this end, recently developed Fokker-Planck-Kolmogorov (FPK) equation approach to probabilistic elasto-plasticity is employed. The most general form of the three dimensional elastic-plastic constitutive rate equation is written in probability density space, resulting in a multi-dimensional FPK equation. The resultant FPK equation is specialized to undrained stress path. Given the second order statistics of the uncertainties in soil properties and assuming Cam Clay elastic-perfectly plastic constitutive model, the specialized FPK equations are solved to obtain second order accurate evolutionary (with pseudotime/ strain) joint probability density function of the stress response. Marginal statistics - marginal probability density function, marginal mean, and marginal standard deviation - of the stress components are then obtained from the multivariate probability density function using standard integration techniques.
\end{abstract}

Keywords: Elasto-plasticity, uncertainty, soils, Cam Clay model, Fokker-Planck-Kolmogorov equation

\section{Introduction}

Soil is a nature provided material and it forms by weathering processes during thousands of years. Due to soil formation process, mechanical properties of the soil are varying from one place to another. Since it is not possible to excavate the entire site and obtain the soil properties at each point, soil properties are usually obtained at a finite number of places (e.g., borehole locations) and they are statistically predicted at other places. Predicting soil properties involves some degree of uncertainty which is known as soil spatial variability $[1,2,3]$. There are also other sources of uncertainty such as measurement uncertainty and transformation relation uncertainty. Measurement uncertainty results from imperfection of instruments, error in registering quantities and human error of test-operators $[4,5,6]$. On the other hand, transformation relation uncertainty is introduced when empirical or other correlation models are used to compute soil properties from field measurements [7]. In order to provide a rational and realistic analysis of a geotechnical system, the effect of these uncertainties should be accounted for in our simulations and designs. In conventional deterministic analyses, these uncertainties and random characteristics of soils are considered by using large factors of safety. However, using large factor of safety not only is not economical, but also it may result in an unsafe design. This is due to the fact that values of factors of safety are the same for a given type of application, such as settlement, regardless of degree of uncertainty involved in its calculation. Since the degree of uncertainty is varying for different conditions, using factors of safety may result in an unreliable design [8]. Therefore, in recent years, geotechnical practice has seen an increasing emphasis on probabilistic simulations/designs.

In the field of geotechnical engineering, probabilistic predictions are typically carried out using Monte Carlo simulation (MCS) technique $[9,10,11,12,13]$. However, this method is computationally very expensive for large scale non- linear elastic-plastic problems. In 2007, Jeremic proposed an alternative simulation approach based on the FokkerPlanck- Kolmogorov (FPK) equation for one-dimensional probabilistic elastic-plastic constitutive simulations of soils [14]. The FPK equations approach utilizes a generic solution proposed by Kavvas to stochastic differential equations with random coefficients and random forcing functions, and rewrites the general form of the elastic-plastic constitutive rate equation in the probability density space in obtaining a FPK equation which describes the evolution of the probability density function (PDF) of stress with pseudo time [15]. Specialization of the FPK equation to any particular elastic-plastic 
constitutive model, followed by solution of the specialized FPK equation provides evolution of the PDF of stress with strain following that constitutive model. Using FPK approach, solution strategies for probabilistic elasto-plasticity were discussed by Sett et al. for both linear and non-linear one-dimensional models [16, 17]. In 2009, Jeremi'c and Sett introduced the concept of probabilistic yielding. They showed that by considering the uncertainty in yield stress, the average (mean) constitutive response and the most probable (mode) constitutive response show nonlinear behavior from the very beginning, even for the simplest elastic-perfectly plastic material model [18]. To make this method more general, Sett and Jeremi'c also discussed the effect of probabilistic yielding on constitutive simulation under cyclic loading [19] . In 2014, Sadrinezhad and Sett extended the one- dimensional framework to a multi-dimensional framework to simulate the probabilistic elastic plastic constitutive behaviour of soils [20,21].

All the above studies have utilized the von Mises plasticity model to simulate the elastic plastic behavior of uncertain soils. However, based on previous studies, elastic plastic models based on critical state formulation that contain features such as hardening, softening and pressure sensitivity typical in soils have been more successful in describing the mechanical behaviors of soils [22, 23, 24]. Therefore, in this study, the Cam Clay plasticity model of critical state soil mechanics, which is one of the most widely used plasticity models, has been used to investigate the influence of uncertainties in soil properties on simulated undrained triaxial constitutive response. The results have been illustrated in terms of marginal statistics - marginal probability density function, marginal mean, and marginal standard deviation - of the stress components, which are obtained from the multivariate probability density function using standard integration techniques.

\section{FPKE Based Probabilistic Elasto-Plasticity in Multi-Dimension}

In multi-dimension, constitutive rate equation becomes a series of simultaneous ordinary differential equations (ODEs), which relates the rates of stress components $(\mathrm{dsi} j=\mathrm{dt}$ ) with the rates of strain components $(\mathrm{dskl}=\mathrm{dt}) \mathrm{using}$ a modulus tensor $(\mathrm{Ci} \mathrm{jkl})$ as follows:

$$
\frac{d \sigma_{i j}}{d t}=C_{i j k l} \frac{d \varepsilon_{k l}}{d t}
$$

where, depending upon whether the stress state is within the yield surface or on the yield surface, the modulus tensor $\left(\mathrm{C}_{\mathrm{ijkl}}\right)$ could be elastic (el) or elastic-plastic (ep):

$$
C_{i j k l}= \begin{cases}C_{i j k l}^{e l} & \text { when el } \\ C_{i j k l}^{e l}-\frac{C_{i j r s}^{e l} \frac{\partial U}{\partial \sigma_{r s}} \frac{\partial f}{\partial \sigma_{p q}} C_{p q k l}^{e l}}{\frac{\partial f}{\partial \sigma_{a b}} C_{a b c d}^{e l} \frac{\partial U}{\partial \sigma_{c d}}-\frac{\partial f}{\partial q_{*}} r_{*}} & \text { when ep }\end{cases}
$$

In the above equation, $\mathrm{C}^{\mathrm{eli}}{ }_{\mathrm{jkl}}, \mathrm{f}, \mathrm{U}, \mathrm{q}_{*}$, and $\mathrm{r}_{*}$ are multiaxial elastic modulus, yield surface, plastic potential surface, internal variable(s), and rate(s) of evolution of internal variable(s), respectively. The tensor of elastic moduli, $\mathrm{C}^{\mathrm{el}}{ }_{\mathrm{ijkl}}, \mathrm{can}^{\mathrm{b}} \mathrm{be}$ expressed in terms of Young's modulus $(E)$, Poisson's ratio (v), and Kronecker delta $(\delta)$ :

$$
C_{i j k l}^{e l}=\frac{E}{2(1+v)}\left[\frac{2 v}{(1-2 v)} \delta_{i j} \delta_{k l}+\delta_{i k} \delta_{j l}+\delta_{i l} \delta_{j k}\right]
$$

Note that if any or all of the above material properties are uncertain, Eq. (1) becomes a set of stochastic differential equation (SDEs) with uncertain coefficients. In such a case, Eq. (1) may be written in the probability density space to obtain a multidimensional FPK equation [17]: 


$$
\begin{aligned}
& \frac{\partial P\left(\sigma_{i j}(t), t\right)}{\partial t}=-\frac{\partial}{\partial \sigma_{m n}}\left[\left\{\left\langle\eta_{m n}\left(\sigma_{m n}(t), C_{m n r s}, \varepsilon_{r s}(t)\right)\right\rangle\right.\right. \\
& \left.+\int_{0}^{t} d \tau \operatorname{Cov}_{0}\left[\frac{\partial \eta_{m n}\left(\sigma_{m n}(t), C_{m n r s}, \varepsilon_{r s}(t)\right)}{\partial \sigma_{a b}} ; \eta_{a b}\left(\sigma_{a b}(t-\tau), C_{a b c d}, \varepsilon_{c d}(t-\tau)\right]\right\} P\left(\sigma_{i j}(t), t\right)\right] \\
& +\frac{\partial^{2}}{\partial \sigma_{m n} \partial \sigma_{a b}}\left[\left\{\int_{0}^{t} d \tau \operatorname{Cov}_{0}\left[\eta_{m n}\left(\sigma_{m n}(t), C_{m n r s}, \varepsilon_{r s}(t)\right) ; \eta_{a b}\left(\sigma_{a b}(t-\tau), C_{a b c d}, \varepsilon_{c d}(t-\tau)\right)\right]\right\}\right. \\
& \left.\quad P\left(\sigma_{i j}(t), t\right)\right]
\end{aligned}
$$

The above equation is an advection-diffusion equation; the terms within the first curly braces constitute the advection coefficient and the terms within the second curly braces constitute the diffusion coefficient. Denoting the advection coefficient by $\mathrm{N}_{(1)}$ and diffusion coefficient by $\mathrm{N}_{(2)}$, Eq. (3) can be written in a more compact form as:

$$
\frac{\partial P\left(\sigma_{i j}, t\right)}{\partial t}=-\frac{\partial}{\partial \sigma_{m n}}\left[N_{(1)_{m n}} P\left(\sigma_{i j}, t\right)-\frac{\partial}{\partial \sigma_{a b}}\left\{N_{(2)_{\text {mnab }}} P\left(\sigma_{i j}, t\right)\right\}\right]
$$

Eq. (4) is the most general form of the FPK equation corresponding to any multi-dimensional constitutive rate equation. In the principal stress space, Eq. (4) simplifies to:

$$
\frac{\partial P\left(\sigma_{k}, t\right)}{\partial t}=-\frac{\partial}{\partial \sigma_{r}}\left[N_{(1)_{r}} P\left(\sigma_{k}, t\right)-\frac{\partial}{\partial \sigma_{s}}\left\{N_{(2)_{r s}} P\left(\sigma_{k}, t\right)\right\}\right]
$$

In Eq. (5), $P\left(\sigma_{k}, t\right)$ is the joint probability density of the principal stresses, $t$ is the pseudo-time, while $N_{(1) r}$ and $N_{(2) r s}$ are the multi-dimensional advection and diffusion coefficients which for elastic isotropic material, take the following forms:

$$
\begin{aligned}
& N_{(1)_{r}}^{e l}=\left\langle C_{r s}^{e l} \frac{d \varepsilon_{r}}{d t}\right\rangle \\
& N_{(2)_{r s}}^{e l}=t \operatorname{Var}\left[C_{r s}^{e l} \frac{d \varepsilon_{r}}{d t}\right]
\end{aligned}
$$

where, $\varepsilon_{\mathrm{i}}$ are the principal strains, $t$ is the pseudo time of the constitutive rate equation (Eq. (1)), and the superscripts ${ }^{. e l}$ on the advection and diffusion coefficients refer to pre-yield elastic region. Furthermore, $\langle\cdot\rangle$ represents the expectation operator, while Var $[\cdot]$ is the variance operator. Hence, given the statistical description (mean and variance) of the Young's modulus and the Poisson's ratio of any material, the governing FPK equation (Eq. (5)) with advection and diffusion coefficients given by Eq. (6) can be solved to obtain its elastic probabilistic constitutive response. For multi dimensional elastic-perfectly plastic constitutive relationship, the advection and diffusion coefficients for post-yield region would be as follows:

$$
\begin{aligned}
& N_{(1)_{r}}^{e l-p l}=\left\langle C_{r s}^{e l-p l} \frac{d \varepsilon_{r}}{d t}\right\rangle \\
& N_{(2)_{r s}}^{e l-p l}=t \operatorname{Var}\left[C_{r s}^{e l-p l} \frac{d \varepsilon_{r}}{d t}\right]
\end{aligned}
$$

in which $\mathrm{C}^{\mathrm{el} \_\mathrm{pl}} \mathrm{rs}$ is the elastic-perfectly plastic tensor which in multi-dimensional space is given by: 


$$
C_{i j k l}^{e l-p l}=C_{i j k l}^{e l}-\frac{C_{i j r s}^{e l} \frac{\partial U}{\partial \sigma_{r s}} \frac{\partial f}{\partial \sigma_{p q}} C_{p q k l}^{e l}}{\frac{\partial f}{\partial \sigma_{a b}} C_{a b c d}^{e l} \frac{\partial U}{\partial \sigma_{c d}}}
$$

Note that the pre-yield advection and diffusion coefficients for the elastic-perfectly plastic model will be of the same forms given by Eq. (6). In reality, however, within a representative volume element (RVE) of a heterogeneous material like soils, each of the large number of particle contacts may have different yield strengths and orientations [25]. In other words, some particle-to-particle slips may occur earlier than the others. Under the framework of probability theory, these possibilities are governed by the probability density function of yield strength $\left(\sigma_{y}\right)$, which can be quantified by statistically analysing constitutive test results. Hence, to realistically simulate the probabilistic material behavior, equivalent advection and diffusion coefficients may be introduced by considering probability weights, based on probability density function of yield strength $\left(\sigma_{y}\right)$, to the elastic and plastic advection and diffusion coefficients [26]. These equivalent coefficients assign probability weights to the stress response based on the probability of material being elastic or elastic-plastic. For elastic plastic material model with uncertain yield strength $\left(\sigma_{\mathrm{y}}\right)$, the equivalent advection and diffusion coefficients $\left(\mathrm{N}^{\mathrm{eq}}(1) \mathrm{r}\right.$ and Neq(2)rs) would become [26]:

$$
\begin{aligned}
& N_{(1)_{r}}^{e q}(\sigma)=\left(1-P\left[\sigma_{y} \leq \sigma\right]\right) N_{(1)_{r}}^{e l}+P\left[\sigma_{y} \leq \sigma\right] N_{(1)_{r}}^{e l-p l} \\
& N_{(2)_{r s}}^{e q}(\sigma)=\left(1-P\left[\sigma_{y} \leq \sigma\right]\right) N_{(2)_{r s}}^{e l}+P\left[\sigma_{y} \leq \sigma\right] N_{(2)_{r s}}^{e l-p l}
\end{aligned}
$$

where $1-P\left[\sigma_{\mathrm{y}} \leq \sigma\right]$ represents the probability of material being elastic and $P\left[\sigma_{\mathrm{y}} \leq \sigma\right]$ represents the probability of material being elastic-plastic. Hence, given the statistical description of Young's modulus, Poisson's ratio, and yield strength of any material, the governing FPK equation (Eq. (5)) with adcevtion and diffusion coefficients given by Eq. (9) can be solved to obtain its elastic-plastic constitutive response. Note that the initial condition of the governing FPK equation (Eq. (5)) can be best represented by a Dirac delta function. For numerical simulation purpose the Dirac delta initial condition may be approximated by a multivariate Gaussian function with a mean of $\mathrm{c}$ and a very small standard deviation $(w)$ as below:

$$
\left.P\left(\sigma_{1}, \sigma_{2}, \sigma_{3}, t\right)\right|_{t=0}=\frac{1}{(2 \pi)^{\frac{3}{2}} \cdot w^{3}} e^{\left[-\frac{1}{2}\left\{\left(\frac{\sigma_{1}-c}{w}\right)^{2}+\left(\frac{\sigma_{2}-c}{w}\right)^{2}+\left(\frac{\sigma_{3}-c}{w}\right)^{2}\right\}\right]}
$$

Reflective boundary conditions are found to be the most appropriate for such FPK equations. They can be mathematically written as:

$$
\left.\zeta_{r}\left(\sigma_{1}, \sigma_{2}, \sigma_{3}, t\right)\right|_{\text {At Boundaries }}=0
$$

where, $\zeta$ is probability current and is given as:

$$
\zeta_{r}=-N_{(1)_{r}} P\left(\sigma_{1}, \sigma_{2}, \sigma_{3}, t\right)+\frac{\partial}{\partial \sigma_{r}}\left\{N_{(2)_{r s}} P\left(\sigma_{1}, \sigma_{2}, \sigma_{3}, t\right)\right\}
$$

\section{Results and Discussions}

In this section, the triaxial FPK PDE (Eq. (5)) is specialized to undrained stress path. Then, by assuming Cam Clay elastic-perfectly plastic constitutive model and appropriate (probabilistic) soil parameters, the specialized FPK PDE is numerically solved to obtain the respective evolutionary joint PDF of stress components with pseudo time/strain. 
Under undrained condition, the soil does not change its volume i.e., volumetric strain remains zero $\left(d \varepsilon_{1}+d \varepsilon_{2}+d \varepsilon_{3}=0\right)$. Further, during an undrained triaxial test, the total confining pressure is constant in the 2 and 3 directions. As a result, the effective stresses in the 2 and 3 directions, $\sigma_{2}$ and $\sigma_{3}$, vary but remain equal $\left(d \sigma_{2}=d \sigma_{3}\right)$. Following Hooke's law and assuming material isotropy, $d \varepsilon_{2}=d \varepsilon_{3}$. Therefore, for a displacement-controlled undrained triaxial simulation with deterministic rate of strain in the 1 direction equal to $\frac{d \varepsilon 1}{d t}$, the rates of strain in the 2 and 3 directions can be calculated as:

$$
\frac{d \varepsilon_{2}}{d t}=\frac{d \varepsilon_{3}}{d t}=-0.5 \frac{d \varepsilon_{1}}{d t}
$$

In this study, the rate of strain in 1 direction is assumed to be 0.5 . Therefore, by assuming triaxial stress condition i.e., $\sigma_{2}=$ $\sigma_{3}$, the rates of strain in the 2 and 3 directions would both be computed to be equal to -0.25 . Using the above rates of strain, Eq. (5) was solved for evolutionary (with pseudo time/strain) joint PDF of $\sigma_{1}$ and $\sigma_{2}$. A probabilistic shear modulus, $G$, given by a Weibull distribution with a mean of $10 \mathrm{MPa}$ and a coefficient of variation (COV) of $50 \%$ was assumed for this simulation. To satisfy the condition of no volume change, the total stress undrained bulk modulus $\mathrm{K}$ must be infinite which implies that the undrained Poisson's ratio must be 0.5. Note that initially all the probability mass should theoretically be concentrated at $\sigma_{r}=\left\{\sigma_{c}, \sigma_{c}, \sigma_{c}\right\}$ which can be mathematically described by a Dirac delta condition. It should be mentioned that $\sigma_{c}$ is the initial confining pressure which assumed to be $100 \mathrm{KPa}$. However, as mentioned earlier, for numerical simulation of FPK equation, Dirac delta initial condition was approximated by a Gaussian function of mean equal to the confining pressure and COV of $10 \%$ as shown in Figure 1. This initial error in standard deviation advected and diffused into the domain during the simulation of the evolution process. Note that this error could be minimized by better approximating the Dirac delta initial condition.

To simulate the elastic plastic constitutive behavior of uncertain soils, Cam Clay plasticity model has been used. The Cam Clay yield limit is usually introduced as an ellipse in the plane of hydrostatic pressure, $p$, and shear equivalent stress, $q$, defined as

$$
\begin{gathered}
p=\sigma_{m}=\frac{\left(\sigma_{1}+\sigma_{2}+\sigma_{3}\right)}{3} \\
q=\sqrt{3 J_{2}}
\end{gathered}
$$

where $J_{2}$ is the second invariant of the stress deviator, respectively. The Cam Clay yield function in terms of $p$ and $q$ can be written as

$$
f=q^{2}-M^{2}\left[p\left(p_{0}-p\right)\right]
$$

where $M$ denotes a material constant which is equal to the slope of the critical state line in the $p-q$ plane and is the locus of points where all ellipses, for any major diameter $p_{0}$, have outward normal parallel to the shear axis. The positive material

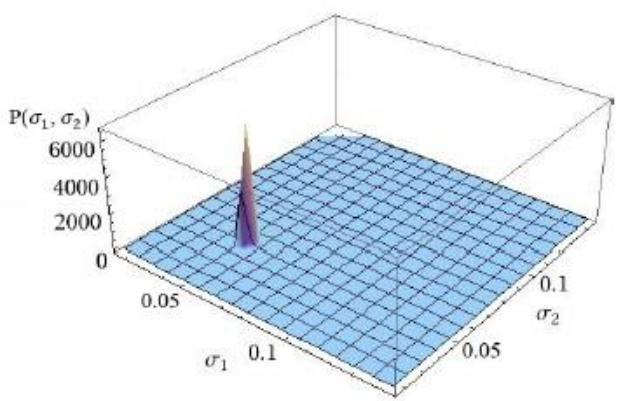

Fig. 1: Simulated probabilistic undrained elastic behavior in terms of joint PDF of $\sigma_{1}$ and $\sigma 2$ at (a) axial strain, $\varepsilon_{\mathrm{a}}\left(\right.$ or $\left.\varepsilon_{1}\right)=0 \%$. 
strength parameter $\mathrm{p}_{0}$ (related to the pre-consolidation pressure) equals the diameter of the ellipse in the direction of the axis of hydrostatic pressure $p$ [27]. For Cam Clay elastic-perfectly plastic material model with uncertain $(M)$, the equivalent advection and diffusion coefficients $\left({ }^{C c} N_{(1)_{r}}^{e q}\right.$ and $\left.{ }^{{ }^{c} c} N_{(2) r s}^{e q}\right)$ take the following forms:

$$
\left\{\begin{array}{l}
{ }^{C c} N_{(1)_{r}}^{e q}(\sigma)=\left(1-P\left[M \leq \sqrt{\frac{q^{2}}{p\left(p_{0}-p\right)}}\right]\right) N_{(1)_{r}}^{e l}+P\left[M \leq \sqrt{\frac{q^{2}}{p\left(p_{0}-p\right)}}\right] N_{(1)_{r}}^{e l-p l} \\
{ }^{C c} N_{(2)_{r s}}^{e q}(\sigma)=\left(1-P\left[M \leq \sqrt{\frac{q^{2}}{p\left(p_{0}-p\right)}}\right]\right) N_{(2)_{r s}}^{e l}+P\left[M \leq \sqrt{\frac{q^{2}}{p\left(p_{0}-p\right)}}\right] N_{(2) r s}^{e l-p l}
\end{array}\right.
$$

For a elastic-perfectly plastic model, ${ }^{N_{(1)_{r}}^{e l-p l}}=N_{(2)_{r s}}^{e l-p l}=0$. Therefore

$$
\left\{\begin{array}{l}
{ }^{C} N_{(1)_{r}}^{e q}(\sigma)=\left(1-P\left[M \leq \sqrt{\frac{q^{2}}{p\left(p_{0}-p\right)}}\right]\right) N_{(1)_{r}}^{e l} \\
{ }^{C} N_{(2)_{r s}}^{e q}(\sigma)=\left(1-P\left[M \leq \sqrt{\frac{q^{2}}{p\left(p_{0}-p\right)}}\right]\right) N_{(2)_{r s}}^{e l}
\end{array}\right.
$$

where $\quad\left(1-P\left[M \leq \sqrt{\frac{q^{2}}{p\left(p_{0}-p\right)}}\right]\right.$ represents the probability of material being elastic and $\left(P\left[M \leq \sqrt{\left.\frac{q^{2}}{p\left(p_{0}-P\right)}\right]}\right)\right.$ represents the probability of material being elastic-plastic. In this study, the material constant for the Cam Clay model, M, was assumed to have a Weibull distribution with a mean of $1.2 \mathrm{MPa}$ and a COV of $10 \%$. p0 was assumed to be deterministic and equal to $0.03 \mathrm{Mpa}$. Figure 2 shows the solution of the governing FPK equation at an axial strain $\left(\varepsilon_{a}\right.$ or $\left.\varepsilon_{1}\right)$ of $0.3 \%$ in terms of joint probability density of $\sigma_{1}$ and $\sigma_{2}$. As can be seen in Figure 2, the joint PDF of $\sigma 1$ and $\sigma 2$ advected and diffused as pseudo time/strain evolved indicating the propagation of uncertainty in shear modulus, $G$.

The evolutionary joint PDF of $\sigma 1$ and $\sigma 2$ was marginalized by standard integration technique and is shown in Figures 3 (a) and (b) in terms of evolutionary marginal PDF of $\sigma l$ and $\sigma 2$, respectively. These figures show how the uncertainty in shear modulus, $G$, affects the PDFs of stress components in the 1 and 2 directions.

The evolutionary, marginal mean and mean \pm standard deviation behaviors of deviatoric stress can also be obtained by post-processing the evolutionary marginal PDFs of $\sigma 1$ and $\sigma 2$; thereby, the response of the soil can be depicted in traditional deviator stress (q) vs. axial strain $\left(\varepsilon_{a}\right)$ and deviator stress $(q)$ vs. trixial shear strain $\left(\varepsilon_{q}\right)$ spaces. Figures 4 (a) and (b) show the results in the above two traditional spaces, but in terms of mean and mean \pm standard deviation behaviors.

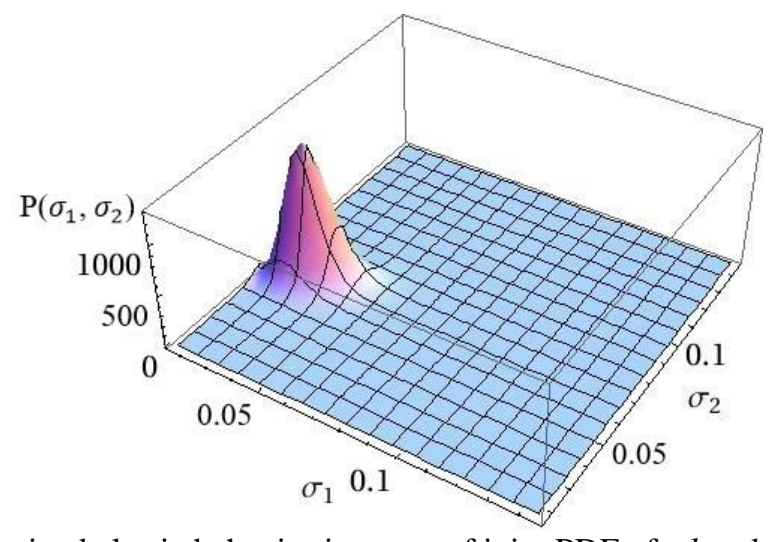

Fig. 2: Simulated probabilistic undrained elastic behavior in terms of joint PDF of $\sigma 1$ and $\sigma 2$ at axial strain, $\varepsilon_{\mathrm{a}}\left(\right.$ or $\left.\varepsilon_{1}\right)=0.3 \%$. 

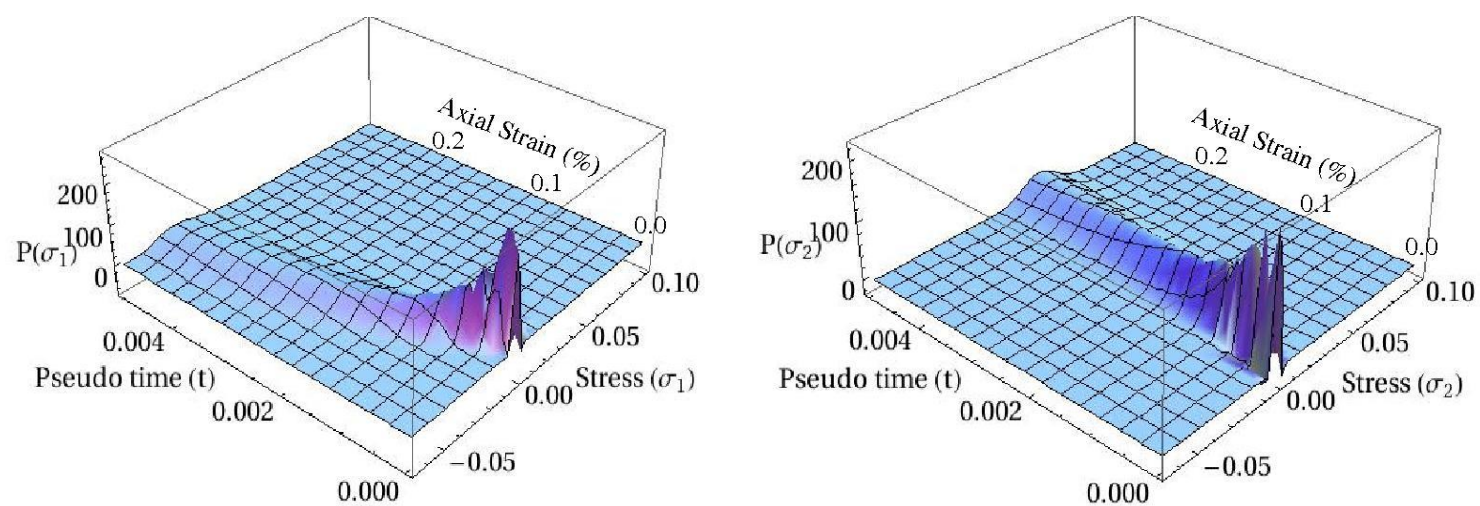

Fig. 3: Simulated probabilistic undrained elastic behavior in terms of evolutionary marginal PDF of (a) $\sigma_{1}$ and (b) $\sigma_{2}$ with pseudo time, $t$.

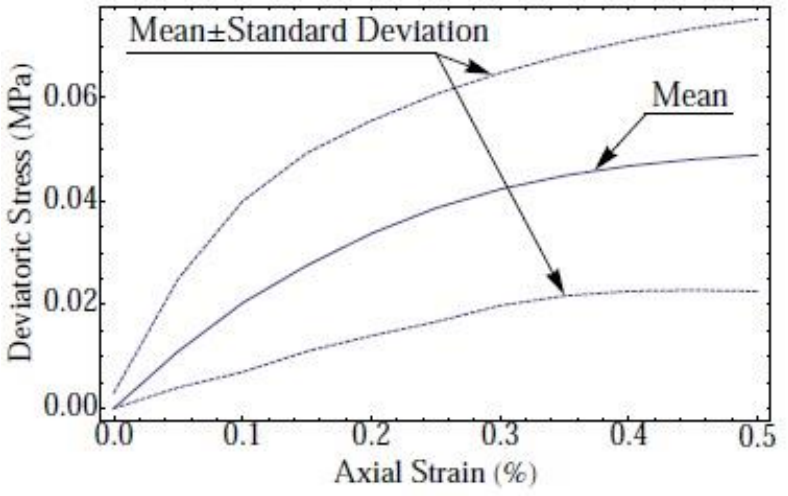

(a)

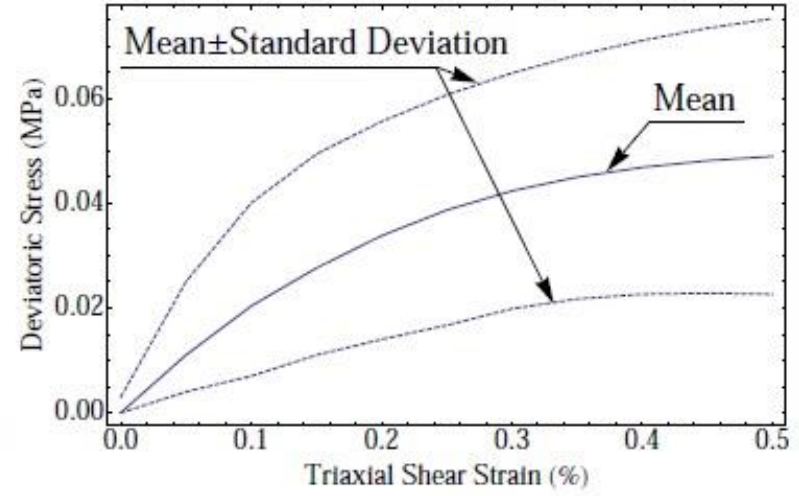

(b)

Fig. 4: Simulated probabilistic undrained elastic-perfectly plastic behavior in terms of (a) deviator stress, $q$ versus axial strain, $\varepsilon_{a}$, and (b) deviator stress, $q$ versus triaxial shear strain, $\varepsilon_{q}$.

It should also be noted that for a conventional undrained trixial compression test in which the cell pressure is held constant while the axial stress is increased, one can write [27],

$$
\delta p=\frac{\delta q}{3}
$$

where $\mathrm{q}$ is the deviator stress and $\mathrm{p}$ is the volumetric stress. Moreover, the principle of effective stress indicates that the excess pore pressure is the difference between the total and effective volumetric stress which in incremental form can be written as [27]:

$$
\delta u=\delta p-\delta p^{\prime}
$$

Since there is no volume change for an undrained constant volume condition, mean effective stress is constant and $\delta p$ ' is zero. Therefore,

$$
\delta u=\delta p=\frac{\delta q}{3}
$$

and hence, the evolution of excess pore water pressure, $u$ can also be computed from the evolutionary behavior of deviatoric stress, $q$. Figure 5 shows the evolutionary excess pore water pressure with trixial shear strain $\left(\varepsilon_{\mathrm{q}}\right)$ for the uncertain elastic soil. 


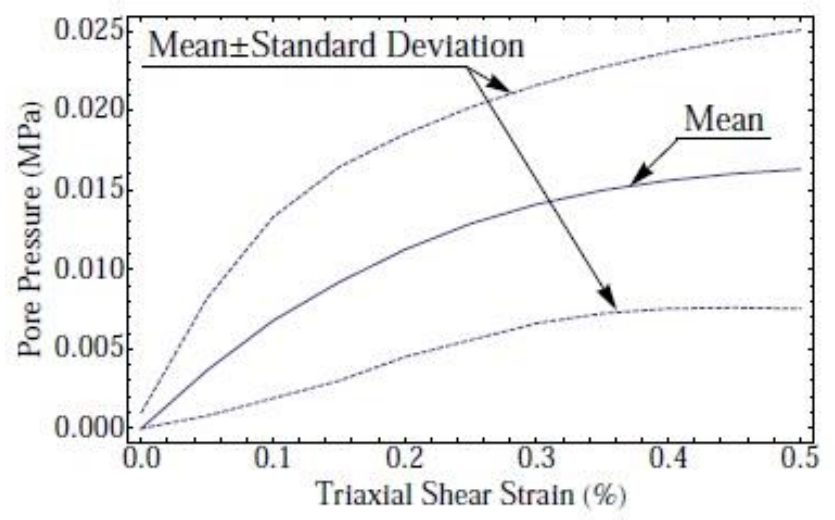

Fig. 5: Simulated probabilistic undrained elastic-perfectly plastic behavior in terms of pore pressure, $u$ versus triaxial shear strain, $\varepsilon_{q}$.

These figures show that, although the material model is elastic-perfectly plastic (i.e, bi-linear), due to uncertainty in yielding, the probabilistic response is smooth, nonlinear and captures the effect of isotropic hardening. This response is very realistic and it means that depending upon the uncertainty in yield strength, there is always a possibility that the soil becomes elasticplastic from the very beginning of loading, which agrees well with the fact that within a RVE of a spatially non-uniform material like soils some particle-to-particle slips may occur earlier than others.

\section{Conclusions}

In this paper, a methodology has been presented for determining the evolution of the joint probability density function of the stress components with the strain components for uncertain elastic-plastic soils. The methodology is based on a multidimensional Fokker-Planck-Kolmogorov equation approach and it utilizes the Cam Clay elastic-perfectly plastic constitutive model. The evolutionary mean and standard deviation of the stress-strain behaviors were obtained by postprocessing the results of the simulations. The evolutionary mean behavior could be explained as the ensemble average of behaviors of all the soil particles in a RVE (soil specimen). On the other hand, the evolutionary standard deviation behavior may be used as an indicator of the dispersion of the response around the mean. The region between the mean and meanstandard deviation, which contained the most probable values of the response, was also obtained. This means that depending upon the uncertainties in the soil properties and interaction between them, there would be a band around the mean response that contained the most probable values of the response.

The results also indicated that, even with the elastic-perfectly plastic (i.e, bi-linear) model, due to uncertainty in yielding, the probabilistic response was smooth and nonlinear from the beginning. This response is very realistic and it means that depending upon the uncertainty in yield strength, there is always a possibility that the soil becomes elasticplastic from the very beginning of loading, which agrees well with the fact that within a RVE of a spatially non-uniform material like soils some particle-to-particle slips may occur earlier than others.

\section{Acknowledgment}

The work presented in this paper is partially supported by grants from NASA/OSGC and US NSF (through award \# CMMI-1200196; cognizant program director: Dr. Richard Fragaszy).

\section{References}

[1] G. A. Fenton, "Estimation of stochastic soil models," Journal of Geotechnical and Geoenvironmental Engineering, ASCE, vol. 125, no. 6, pp. 470-485, 1999.

[2] A. F. Gordon, "Random field modeling of CPT data," Journal of Geotechnical and Geoenvironmental Engineering, ASCE, vol. 125, no. 6, pp. 486-498, 1999.

[3] D. J. DeGroot and G. B. Baecher, "Estimating autocovariance of in-situ soil properties," Journal of Geotechnical Engineering, vol. 119, no. 1, pp. 147-166, 1993.

[4] G. M. Hammitt, "Statistical analysis of data from a comparative laboratory test program sponsored by ACITL," U.S. Army Waterways Experiment Station, Vicksburg, MS, 1966. 
[5] K.-K. Phoon and F. H. Kulhawy, "Characterization of geotechnical variability," Canadian Geotechnical Journal, vol. 36, no. 4, pp. 612-624, 1999.

[6] K. T. Marosi and D. R. Hiltunen, "Characterization of spectral analysis of surface waves shear wave velocity measurement uncertainty," Journal of Geotechnical and Geoenvironmental Engineering, ASCE, vol. 130, no. 10, pp. 1034-1041, 2004.

[7] K.-K. Phoon and F. H. Kulhawy, "Evaluation of geotechnical property variability," Canadian Geotechnical Journal, vol. 36, no. 4, pp. 625-639, 1999.

[8] J. M. Duncan, "Factors of safety and reliability in geotechnical engineering," Journal of Geotechnical and Geoenvironmental Engineering, ASCE, vol. 126, no. 4, pp. 307-316, 2000.

[9] G. M. Paice, D. V. Griffiths, and G. A. Fenton, "Finite element modeling of settlement on spatially random soil," Journal of Geotechnical Engineering, vol. 122, no. 9, pp. 777-779, 1996.

[10] R. Popescu, J. H. Prevost, and G. Deodatis, "Effects of spatial variability on soil liquefaction: Some design recommendations," Geotechnique, vol. 47, no. 5, pp. 1019-1036, 1997.

[11] B. S. L. P. De Lima, E. C. Teixeira, and N. F. F. Ebecken, "Probabilistic and possibilistic methods for the elastoplastic analysis of soils," Advances in Engineering Software, vol. 132, pp. 569-585, 2001.

[12] S. Koutsourelakis, J. H. Prevost, and G. Deodatis, "Risk assesment of an interacting structure-soil system due to liquefaction," Earthquake Engineering and Structural Dynamics, vol. 31, pp. 851-879, 2002.

[13] D. V. Griffiths, G. A. Fenton, and N. Manoharan, "Bearing capacity of rough rigid strip footing on cohesive soil: Probabilistic study," Journal of Geotechnical and Geoenvironmental Engineering, ASCE, vol. 128, no. 9, pp. 743$755,2002$.

[14] B. Jeremic, K. Sett, and M. L. Kavvas, "Probabilistic elasto-plasticity: Formulation in 1-D," Acta Geotechnica, vol. 2, no. 3, pp. 197-210, 2007.

[15] M. L. Kavvas, "Nonlinear hydrologic processes: Conservation equations for determining their means and probability distributions," Journal of Hydrologic Engineering, vol. 8, no. 2, pp. 44-53, 2003.

[16] K. Sett, B. Jeremic, and M. L. Kavvas, "Probabilistic elasto-plasticity: Solution and verification in 1-D," Acta Geotechnica, vol. 2, no. 3, pp. 211-220, 2007.

[17] K. Sett, B.Jeremic, and M. L. Kavvas, "The role of nonlinear hardening/softening in probabilistic elasto-plasticity," International Journal for Numerical and Analytical Methods in Geomechanics, vol. 31, no. 7, pp. 953-975, 2007.

[18] B. Jeremic and K. Sett, "On probabilistic yielding of materials," Communications in Numerical Methods in Engineering, vol. 25, no. 3, pp. 291-300, 2009.

[19] K. Sett and B. Jeremic, "Probabilistic yielding and cyclic behavior of geomaterials," International Journal for Numerical and Analytical Methods in Geomechanics, vol. 34, no. 15, pp. 1541-1559, 2010.

[20] A. Sadrinezhad and K. Sett, "Multiaxial probabilistic elasticplastic constitutive simulations of soils," Safety, Reliability, Risk and Life-Cycle Performance of Structures and Infrastructures, p. 859866, 2014.

[21] A. Sadrinezhad, K. Sett, and S. Hariharan, "Probabilistic simulation of triaxial undrained cyclic behavior of soils," International Journal of Environmental, Chemical, Ecological, Geological and Geophysical Engineering, vol. 10, no. 4, pp. 380-386, 2016.

[22] J. Atkinson, Foundations and Slopes: An Introduction to Applications of Critical State Soil Mechanics, ser. A Halsted Press Book. Wiley, 1981, [Online]. Available: https://books.google.com/books?id= sFRAAAAMAAJ

[23] M. Zytynski, M. Randolph, R. Nova, and C. Worth, "On modelling the unloading-reloading behaviour of soils," International Journal for Numerical and Analytical Methods in Geomechanics, p. 8793, 1978.

[24] R. I. Borja, "Cam-clay plasticity, part ii: Implicit integration of constitutive equation based a nonlinear elastic stress predictor," Comput. Methods Appl. Mech. Eng., vol. 88, no. 2, pp. 225-240, 1991

[25] Z. Hashin, "Analysis of composite materials," Journal of Applied Mechanics, vol. 50, pp. 481-501, 1983.

[26] B. Jeremic and K. Sett, "On probabilistic yielding of materials," Communications in Numerical Methods in Engineering, vol. 25, no. 3, pp. 291-300, 2009.

[27] D. M. Wood, Soil Behaviour and Critical State Soil Mechanics. Cambridge CB2 8RU, UK: Cambridge University Press, 1990, ISBN: 978-0-521-33782-3. 